Hautarzt 2021 · 72:842

https://doi.org/10.1007/s00105-021-04878-2

Online publiziert: 23. August 2021

๑) Springer Medizin Verlag $\mathrm{GmbH}$, ein Teil von

Springer Nature 2021

\section{Erratum zu: Klinische, pathologische und molekulare Biomarker der Hidradenitis suppurativa/Acne inversa}

\author{
M. von Laffert ${ }^{1,2} \cdot$ R. E. Hunger ${ }^{3} \cdot$ A. A. Navarini ${ }^{4} \cdot$ C. C. Zouboulis ${ }^{1,5}$ \\ 'Hidradenitis Suppurativa Foundation e. V., Dessau, Deutschland \\ ${ }^{2}$ Institut für Pathologie, Campus Charité Mitte, Charité-Universitätsmedizin Berlin, Berlin, Deutschland \\ ${ }^{3}$ Universitätsklinik für Dermatologie, Inselspital - Universitätsspital Bern, Bern, Schweiz \\ ${ }^{4}$ Klinik für Dermatologie, Universitätsspital Basel, Basel, Schweiz \\ ${ }^{5}$ Hochschulklinik für Dermatologie, Venerologie und Allergologie, Immunologisches Zentrum, Städtisches \\ Klinikum Dessau, Medizinische Hochschule Brandenburg Theodor Fontane und Fakultät für \\ Gesundheitswissenschaften Brandenburg, Dessau, Deutschland
}

\section{Erratum zu:}

Hautarzt 2021

https://doi.org/10.1007/s00105-021-

04848-8

In diesem Leitthemenbeitrag war die Affiliation des Autors R.E. Hunger leider fehlerhaft und wurde korrigiert. Wir bitten um Beachtung.

\section{Korrespondenzadresse}

Univ.-Prof. Dr. med. Prof. honoraire Dr. h.c. C. C. Zouboulis

Hochschulklinik für Dermatologie, Venerologie und Allergologie, Immunologisches Zentrum, Städtisches Klinikum Dessau, Medizinische Hochschule Brandenburg Theodor Fontane und Fakultät für Gesundheitswissenschaften Brandenburg

Auenweg 38, 06847 Dessau, Deutschland christos.zouboulis@mhb-fontane.de
Die Online-Version des Originalartikels ist unter https://doi.org/10.1007/s00105-021-04848-8 zu finden.

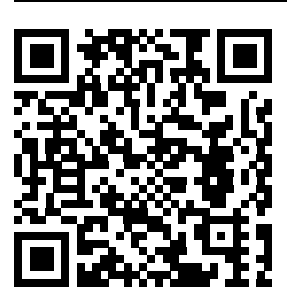

QR-Code scannen \& Beitrag online lesen 